SHORT REPORT

\title{
Postinfectious vasculopathy with evolution to moyamoya syndrome
}

\author{
T Czartoski, D Hallam, J M Lacy, M R Chun, K Becker
}

J Neurol Neurosurg Psychiatry 2005;76:256-259. doi: 10.1136/jnnp.2004.041046

Background: Parainfectious vascular events are a known complication of bacterial meningitis, typically occurring within two weeks of disease onset. Delayed vascular complications are rare. We present a case of progressive vasculopathy following bacterial meningitis.

Case description: A 20 year old woman developed progressive vasculopathy after successful treatment of pneumococcal meningitis. Within eight months of her infection, angiography revealed the appearance of moyamoya syndrome. Despite aggressive immunomodulation and anticoagulation, she had multiple strokes. Autopsy confirmed severe narrowing of proximal cerebral vasculature with absence of inflammation or atherosclerosis.

Conclusions: The inflammation and subsequent postinfectious autoimmune response associated with meningitis can lead to a progressive vasculopathy and may represent a pathophysiologic mechanism for the arterial occlusions seen in moyamoya syndrome.

$\mathrm{V}$ ascular events related to meningitis typically occur within the first two weeks of disease and are most commonly seen with Streptococcus pneumoniae, Mycobacterium tuberculosis, Neisseria meningitidis, and Haemophilus influenzae. $^{1-3}$ Delayed postinfectious vascular events are less commonly reported in association with flow defects from fixed stenoses. ${ }^{45}$ We report a case of postinfectious vasculopathy following pneumococcal meningitis with progression to moyamoya syndrome.

\section{CASE HISTORY}

In July 2002, a 20 year old white woman with a history of migraines and bipolar affective disorder presented with 24 hours of fever, headache, and obtundation. Lumbar puncture (LP) was not performed due to marked cerebral oedema. She was treated empirically with vancomycin, ceftriaxone, and aciclovir. S. pneumoniae sensitive to ceftriaxone was cultured from her blood; ceftriaxone alone was continued for a total of 15 days. On day 9, she developed left sided weakness and a right third nerve palsy. Imaging studies revealed pontine and frontal lobe infarcts. The cerebrospinal fluid (CSF) was consistent with partially treated bacterial meningitis, containing 400 white blood cells (WBCs) with a polymorphonuclear predominance (table 1). Angiography was consistent with vasculitis (fig lA). She was treated with dexamethasone $4 \mathrm{mg}$ every six hours for three days followed by prednisone $60 \mathrm{mg}$ twice daily, with a rapid taper over the course of four weeks.

The steroid taper was completed on day 45 of her illness. On day 48 , she returned to the emergency room (ER) with severe headache. LP revealed 92 WBCs, with a lymphocytic predominance; opening pressure $>56 \mathrm{~cm} \mathrm{H}_{2} \mathrm{O}$. She was started on acetazolamide and her headache resolved the following day. Magnetic resonance imaging (MRI) showed evolution of the previously noted infarcts.

On day 118, the patient was brought to the ER by her family who noted language problems for approximately two weeks. Examination revealed aphasia; MRI showed new infarcts in the left temporal and occipital lobes. CSF was notable for 22 WBCs, $96 \%$ of which were lymphocytes. An angiogram showed progressive stenoses in the anterior and posterior circulation. Anticardiolipin antibodies were not detected, but anti- $\beta 2$-glycoprotein 1 IgG ( $\beta 2$-GPl G) titres were elevated at 29 SGU (reference: 0-9). She was treated with intravenous methylprednisolone (1 gm daily for three days) followed by prednisone $60 \mathrm{mg}$ daily. Anticoagulation, initially with heparin and subsequently with warfarin, was started. An exhaustive search for other causes of cerebrovascular disease was unrevealing.

On day 139, she was admitted with increased agitation and visual disturbance. Examination was remarkable for cortical blindness. MRI revealed a new right occipital infarct (fig 1B). CSF examination was normal. Repeat angiogram on day 150 showed progression of the vascular stenoses (fig 1B). Anti$\beta 2-G P 1 G$ titres had increased to $56 \mathrm{SGU}$; there was no evidence of a lupus anticoagulant. A trial of intra-arterial papaverine failed to change the calibre of the vessels and improve blood flow. Renal angiography revealed arterial changes within the parenchymal vessels consistent with vasculitis. She was treated with cyclophosphamide on day 165 .

Serial transcranial Doppler (TCD) studies were consistent with intracranial stenoses and on day 180 suggested improvement; the anti- $\beta 2-G P 1$ G titres had decreased to 24 SGU. The family declined further therapy with cyclophosphamide due to concerns over side effects. She was discharged on prednisone, $60 \mathrm{mg}$ daily, tapered off over eight weeks; the taper concluded on day 228. Routine outpatient follow up two days later revealed no acute problems. The patient was ambulatory with a mild left hemiparesis and the ability to detect light and motion. On day 237 her carer brought her to the ER because of a change in behaviour and complete blindness. MRI revealed new infarcts in a watershed distribution in the right hemisphere and in the posterior parietal lobe in the left hemisphere (fig IC). An LP revealed a recrudescence of CSF inflammation with 16 WBCs, predominantly lymphocytes. TCD suggested worsening intracranial disease, which was confirmed by angiography. Angiography also showed the development of small collateral vessels in the lenticulostriate distribution reminiscent of early moyamoya disease (fig lC). Anti- $\beta 2-G P 1$ G titres had decreased to 24 SGU. She was again treated with cyclophosphamide $(950 \mathrm{mg})$ and intravenous methylprednisolone ( 1 gm daily for three days) followed by a prednisone taper (starting at $60 \mathrm{mg}$ daily).

Abbreviations: $\beta 2$-GP1, $\beta 2$-glycoprotein 1; CSF, cerebrospinal fluid; LP, lumbar puncture; TCD, transcranial Doppler; WBC, white blood cell 
Table 1 Course of illness, therapeutic interventions, and response to therapy

\begin{tabular}{|c|c|c|c|c|c|c|c|c|c|c|}
\hline Day & 11 & 48 & 55 & 118 & 150 & 158 & 165 & 180 & 230 & 237 \\
\hline Therapy & CTX, ST & - & - & ST & ST & $\mathrm{ST}, \mathrm{AC}$ & $\mathrm{ST}, \mathrm{AC}, \mathrm{CY}$ & $\mathrm{ST}, \mathrm{AC}, \mathrm{CY}$ & $A C$ & $\mathrm{AC}, \mathrm{ST}, \mathrm{CY}$ \\
\hline \multicolumn{11}{|l|}{ Cerebrospinal fluid findings } \\
\hline Red blood cells (per ml) & 7 & 2 & 2 & - & 17 & & & & & 1 \\
\hline White blood cells (per ml) & 400 & 92 & 118 & 22 & 4 & & & & & 16 \\
\hline Polymorphonuclear cells (\%) & 74 & 12 & 16 & 1 & - & & & & & 0 \\
\hline Lymphocytes (\%) & 19 & 73 & 70 & 96 & - & & & & & 94 \\
\hline Monocytes (\%) & 7 & 15 & 14 & 3 & 100 & & & & & 6 \\
\hline Protein (mg/dl) & 224 & 96 & 100 & 84 & 27 & & & & & 43 \\
\hline Glucose (mg/dl) & 56 & 45 & 40 & 56 & 67 & & & & & 72 \\
\hline \multicolumn{11}{|l|}{ Anti-phospholipid antibodies } \\
\hline Anti- $\beta 2$-glycoprotein 1 (lgG; SGU) reference range: 0-9 & & & & 29 & 56 & & & 24 & 52 & \\
\hline \multicolumn{11}{|l|}{ TCD mean flow velocities $(\mathrm{cm} / \mathrm{sec})$} \\
\hline Right MCA, reference velocity: 58 & & & & 164 & 312 & 157 & 287 & 195 & & 350 \\
\hline Left MCA, reference velocity: 58 & & & & 309 & 337 & 334 & 344 & 328 & & $\mathrm{NI}$ \\
\hline Right VA, reference velocity: 45 & & & & 193 & 245 & 105 & 107 & 91 & & 64 \\
\hline Left VA, reference velocity: 45 & & & & 231 & 121 & 140 & 108 & 176 & & 169 \\
\hline Basilar artery, reference velocity: 38 & & & & $\mathrm{NI}$ & 162 & 119 & 193 & 114 & & 136 \\
\hline
\end{tabular}

AC, anticoagulation; CSF, cerebrospinal fluid; CTX, ceftriaxone; CY, cyclophosphamide; NI, not insonated; ST, steroids; TCD, transcranial Doppler ultrasound.

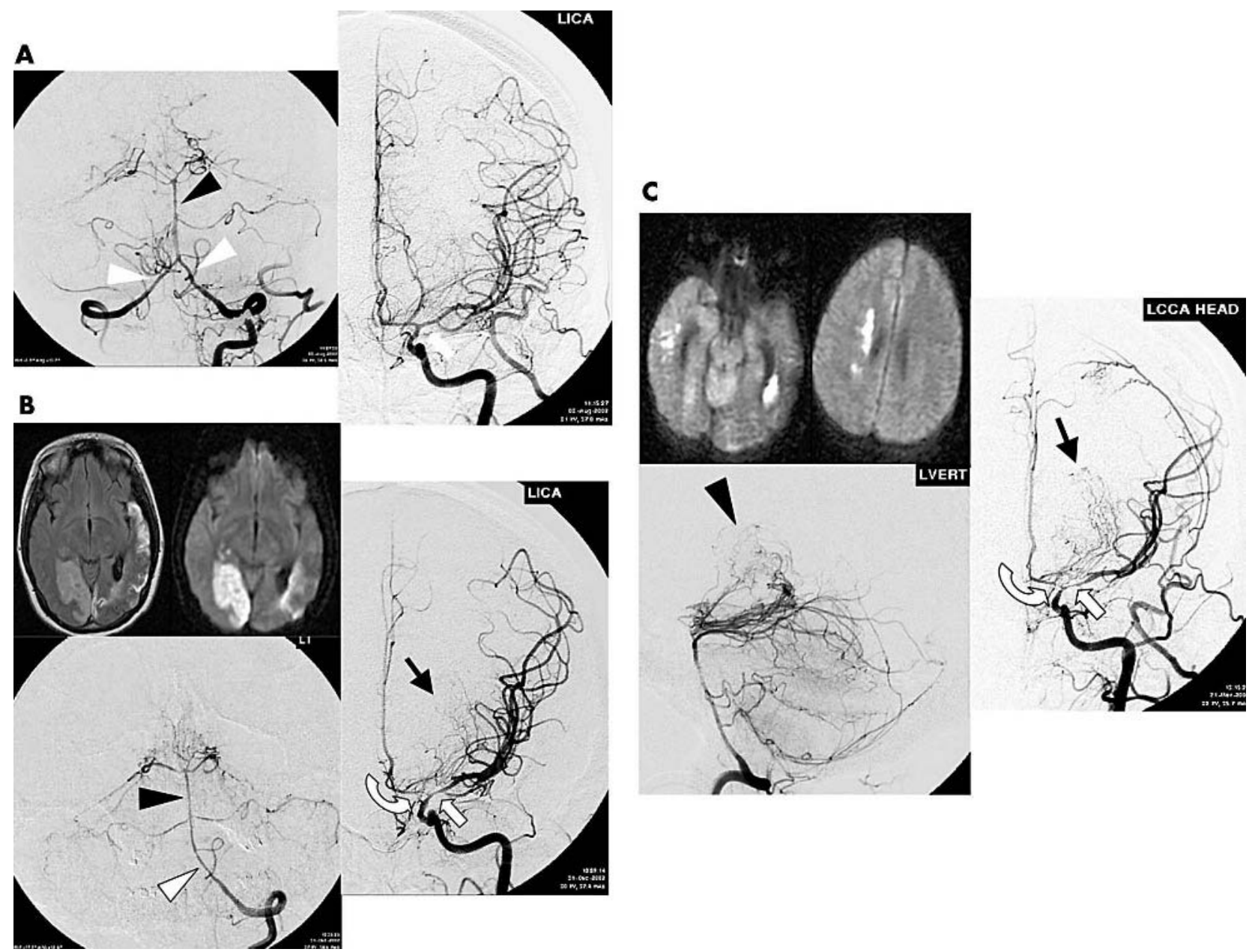

Figure 1 Imaging demonstrates progressive vasculopathy with corresponding infarcts in multiple vascular distributions. Computed tomography (CT) scan 10 days after presentation with meningitis was unremarkable (not shown). (A) Angiogram performed at day 12. The left vertebral injection demonstrates moderate narrowing of distal bilateral vertebral arteries (white arrowheads) and basilar artery (black arrowhead). The left carotid angiogram shows mild narrowing of the M1 segment of the left middle cerebral artery (MCA) (white arrow). (B) On day 139, fluid attenuation inversion recovery (FLAIR) and diffusion weighted images (DWI) show acute right posterior cerebral artery and left MCA distribution infarcts. On day 150, the angiogram shows severe narrowing in the basilar artery (black arrowhead) and left vertebral artery (white arrowhead). The carotid angiogram shows marked progression of the left $M 1$ segment narrowing (straight white arrow) and new stenosis of the $A 1$ segment of the left anterior cerebral artery (ACA) (curved white arrow). At this point, lenticulostriate collaterals become apparent (black arrow). (C) On day 237, DWI confirms acute infarcts in bilateral cerebral hemispheres. Proliferation of posterior penetrating vessels is shown on lateral vertebral angiogram (black arrowhead). There is further proliferation of lenticulostriate collaterals (black arrow), as well as the previously noted severe narrowing of proximal MCA (straight white arrow) and ACA (white curved arrow). 
On day 260, she was brought to the ER by her family with acute obtundation following three days of decreased nutritional intake. The international normalised ratio was 6.3. A head CT scan revealed a large left basal ganglion haemorrhage. She progressed to herniation and expired. An autopsy revealed no systemic vasculitis or arteriopathy. The proximal cerebral vasculature exhibited severe luminal narrowing in the absence of inflammation or atherosclerosis (fig 2).

\section{DISCUSSION}

Vascular events are a known complication of bacterial meningitis but are usually limited to the parainfectious period. $^{1-3}$ This case of postinfectious, progressive vasculopathy following pneumococcal meningitis appears to be the result of a delayed, dynamic process. A recent report by Palacio et al described a patient with late developing intracranial stenoses and benign course following $H$. influenzae meningitis. ${ }^{6}$ Their patient developed headache and transient neurological symptoms six weeks after hospitalisation. Angiograms performed at 2, 9, and 31 months posthospitalisation demonstrated progression followed by improvement of multifocal, intracranial stenoses. In our case, early postinfectious vasculopathy was similarly complicated by a delayed, progressive intracranial vasculopathy with a more malignant course.

The pathogenesis of this type of postinfectious vasculopathy is unknown, although infection could trigger an autoimmune process towards the cerebral blood vessels. Streptococcal infections are associated with a variety of autoimmune diseases, including glomerulonephritis, chorea,

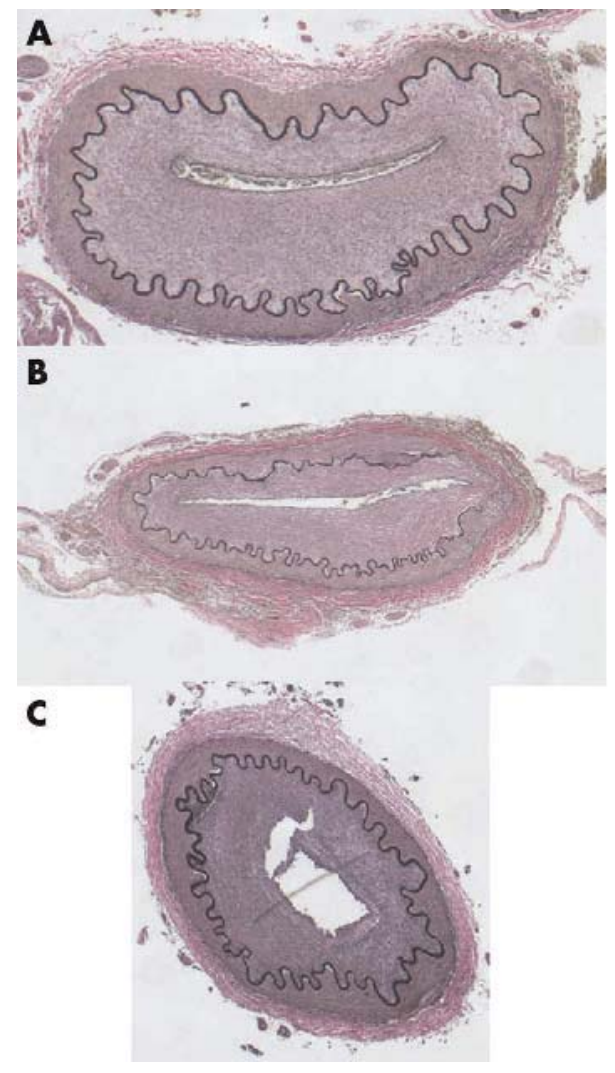

Figure 2 (A) Basilar artery, (B) right vertebral artery, and (C) left middle cerebral artery. The intima is markedly hyperplastic and contains a mixture of fibroblasts, smooth muscle, and collagen. There is hypercrenulation of the internal elastic laminae with focal disruption, duplication and thinning of the media. The adventitia is unremarkable. Inflammation and atherosclerosis are absent (Verhoeff-Van Gieson stain, original magnification $\times 40$ ). myocarditis, arthritis, tics, and obsessive compulsive disorder. ${ }^{78}$ Triggering of antiphospholipid antibody syndromes (APAS) is also reported following infections, and antibodies to $\beta_{2}$-GP 1 are associated with postinfectious autoimmune APAS. ${ }^{9}$ In our patient, $\beta_{2}$-GP 1 levels were elevated during periods of vascular disease progression, which might indicate an aberrant immune response to infection resulting in increased susceptibility to stenosis and thrombosis.

The pathogenesis of moyamoya disease is not understood. Genetic, autoimmune, and infectious aetiologies have been postulated. Autopsy studies of patients with moyamoya disease classically show intimal thickening with crenulation of the elastic lamina-inflammation is absent. ${ }^{10}{ }^{11}$ Similar changes were seen in our patient. The absence of inflammation of the cerebral vessels at autopsy implies that the patient either responded to the aggressive immunomodulatory therapy or the autoimmune process spontaneously arrested.

Unfortunately, no vascular studies were done prior to the onset of the patient's symptoms, so the possibility of preexisting vascular disease cannot be excluded. Several pieces of evidence, however, suggest that her vascular disease was related to the meningitis. First, she was asymptomatic prior to the event. Secondly, she developed progressive stenoses with multiple infarcts over the course of her illness. Thirdly, vessels outside the subarachnoid space were spared, suggesting the vasculopathy resulted from intracranial inflammation, as is seen in meningitis.

This case illustrates the occurrence of postinfectious vasculopathy with progression to moyamoya syndrome following S. pneumoniae meningitis. Due to the progressive course, elevated anti- $\beta_{2}$-GP 1 titres, and transient response to immunomodulatory therapy, we believe that the vasculopathy was mediated by an autoimmune process and suggests a possible pathogenesis for moyamoya syndrome.

\section{ACKNOWLEDGEMENTS}

We would like to thank William T Longstreth Jr and Christina Marra for their helpful comments.

\section{Authors' affiliations \\ T Czartoski, K Becker, Department of Neurology, University of Washington School of Medicine, Harborview Medical Center, Seattle, WA, USA \\ D Hallam, Department of Radiology, University of Washington School of Medicine, Harborview Medical Center, Seattle, WA, USA \\ J M Lacy, Department of Pathology, University of Washington School of Medicine, Harborview Medical Center, Seattle, WA, USA \\ M R Chun, Everett Neurological Center, Everett, WA, USA}

KB is supported by KO2 NS02160-01 NST.

Competing interests: none declared

Correspondence to: Dr K J Becker, Box 359775 Harborview Medical Center, 325 9th Ave, Seattle, WA 98104-249, USA; kjb@u. washington.edu

Received 5 March 2004

In revised form 18 April 2004

Accepted 21 May 2004

\section{REFERENCES}

1 Weststrate W, Hijdra A, deGans J. Brain infarcts in adults with bacterial meningitis. Lancet 1996;347:399.

2 Pfister HW, Borasio GD, Dirnagl U, et al. Cerebrovascular complications of bacterial meningitis in adults. Neurology 1992;42:1497-504.

3 Igarashi M, Gilmartin R, Gerald B, et al. Cerebral arteritis and bacterial meningitis. Arch Neurol 1984;41:531-35.

4 Kerr L, Filloux F. Cerebral infarction as a remote complication of childhood Haemophilus influenzae meningitis. West J Med 1992;157:179-82.

5 Yamashima T, Kashihara K, Ikeda K, et al. Three phases of cerebral arteriopathy in meningitis: vasospasm and vasodilatation followed by organic stenosis. Neurosurgery 1985;16:546-53. 
6 Palacio S, Hart RG, Vollmer DG, et al. Late-developing cerebral arteropathy after pyogenic meningitis. Arch Neurol 2003;60:431-33

7 Cunningham MW. Pathogenesis of group A streptococcal infections. Clin Microbiol Rev 2000;13:470-511.

8 Snider L, Swedo S. Post-streptococcal autoimmune disorders of the central nervous system. Curr Opin Neurol 2003;16:359-65.
9 Asherson R, Cervera R. Antiphospholipid antibodies and infections. Ann Rheum Dis 2003;62:388-93.

10 Hosoda Y, Ikeda E, Hirose S. Histopathological studies on spontaneous occlusion of the circle of Willis (cerebrovascular moyamoya disease). Clin Neurol Neurosurg 1997:99(suppl 2):s203-s208.

11 Fukui M, Kono S, Sueishi K, et al. Moyamoya disease. Neuropathology 2000;20:s61-s64.

\section{HISTORICAL NOTE}

\section{Brodmann's cortical maps}

V icq d'Azyr, a physician and artist, described the brain's convolutions in 1786, noting differences in morphology in other animals. Magendie had written similarly.

Early attempts to correlate the cerebral anatomy to function by observed neurological deficits began in the 1820s, the result of the work of Franz Gall, ${ }^{1}$ Bouillaud, Robert Todd, Rolando, and many others (references in). ${ }^{2}$ Pierre Gratiolet and Francois Leuret mapped the folds and fissures of the cerebral cortex, and named the frontal, temporal, parietal, and occipital lobes.

It was Korbinian Brodmann (1868-1918) who refined nomenclature by numbering discrete cortical areas in mapsscorned with arguable justification by Henry Head.

Brodmann was born at Liggersdorf, Hohenzollern. He qualified at Freiburg in 1895. An attack of diphtheria halted his progress but he became Assistant to the Neurological Clinic at Alexanderbad im Fichtelberg, whose chief was the famed and influential Oskar Vogt, who in 1898 created the Neurobiologisches Universitäts-Laboratorium in Berlin. Brodmann briefly followed Vogt to Berlin and then studied pathology in Leipzig, obtaining his MD in 1898 with the thesis: A contribution to the understanding of chronic ependymal sclerosis. He worked at the University Psychiatric Clinic in Jena, under Binswanger and later at Frankfurt-am Main from 1900 to 1901. There he was stimulated by Carl Weigert, who was developing cellular staining techniques, and by Nissl, Edinger, and Alzheimer-major influences. In late 1901 Brodmann returned to Vogt in Berlin where he met Bielschowsky, who developed silver impregnation of nerve fibres. Between 1903 and 1908 he published seven papers"; many concerned with the comparative cytoarchitectonics of mammalian cortex. Vogt suggested to Brodmann that he systematically studied the cells of the cerebral cortex, using sections stained with the new method of Franz Nissl (18601919). However, the Berlin Medical Faculty rejected his "habilitation" thesis on the pro-simian cortex and his major research was performed despite serious lack of funding from Berlin University.

He described the different cytoarchitectonic structure of the gyri and showed that the human cortex is organised anatomically in the same way in man and other mammals. The cortex consisted of six layers, and, on this basis, he devised his numeric system for referring to different cortical areas. This culminated in his magnum opus in 1909, Vergleichende lokalisationslehre der grosshirnrinde (localisation in the cerebral hemispheres: a comparative study), which described 52 discrete cortical areas. He defined cytoarchitectonics as

"The localization of the individual histological elements, their layering, and their parcellation in the adult brain."

Although there were other investigators of cytoarchitectonics, ${ }^{78}$ Brodmann's maps and, especially, his numerical system attached to cytoarchitectonics were widely accepted; they were his major contribution. He had studied 64 different mammalian species. However, he faced difficulties, observing:

doi: 10.1136/jnnp.2004.037200

"First and foremost we still lack clear criteria for the recognition of anatomically equivalent cellular elements...There has been occasional talk of 'sensory cells' located in particular regions, or of sensorial 'special cells'. People have invented acoustic or optical special cells and even a 'memory' cell, and have not shied away from the fantastic 'psychic cell'."

This, he stoutly rejected, concluding, "Functional localisation without the lead of anatomy is utterly impossible."

Oskar Vogt described Brodmann as having "broad scientific interests, a good gift of observation and great diligence in widening his knowledge". His interests extended to neurology, psychiatry, physiology, zoology, and anthropology. He was described as "an intense and earnest man, reserved to the point of timidity, but could flare, on occasion, into a temper", 9 possibly frustrated by his inability to secure a permanent job. Not until 1916, aged 48, did Brodmann obtain this security. He had left Berlin in 1910 to work with Gaupp at Tübingen, where he was made titular professor in 1913. Finally, in 1918, he accepted an invitation from Munich to take the Chair of the Topographical Histological Department at the research centre for psychiatry. He died in $1918^{10}$ of septicaemia complicating pneumonia.

In 1919, Cecile Vogt described over 200 cortical areas and six years later von Economo and Koskinas revised the nomenclature.

J M S Pearce 304 Beverley Road, Anlaby, East Yorkshire HU10 7BG, UK; jmspearce@freenet.co.uk

\section{References}

1 Gall F. Sur les fonctions du cerveau et sur celles de chacune de ses parties. Paris: Schoell, 1822-25.

2 Pearce JMS. In: Fragments of neurological history. London: Imperial College Press, 2003:14-31.

3 http://www.korbinian-brodmann.de/english/brodmann.html.

4 Haymaker W, Schiller F, eds. The founders of neurology, 2nd edn. Springfield, Illinois: Charles C Thomas, 1970.

5 Bielchowsky M, Brodmann K. Zur feineren histologie und histopathologie der grosshirnrinde mit besonderer berücksichtigung der dementia paralytica, dementia senilis und idiotie. Journal für Psychologie und Neurologie 1905;5:173-99.

6 Brodmann K. Vergleichende localisationslehre der grosshirnrinde in ihren principien dargestellt auf grund des zellenbaues. Leipzig: Barth, 1909, [Trans Garey L. Brodmann's localisation in the Cerebral Cortex. London: SmithGordon, 1994].

7 Kemper T, Le Brun T, Galaburda AM. Principles of cytoarchitectonics. In: Peters A, Jones EG, eds. Cerebral Cortex. New York: Plenum, 1984:35-57.

8 Smith CUM. A century of cortical architectonics. Journal of the History of Neuroscience 1992;1:201-18.

9 Rose JE. Korbinian Brodmann. In: Haymaker W, Schiller F, eds. The founders of neurology, 2nd edn. Springfield, Illinois: Charles C Thomas, 1970.

10 Vogt O. Korbinian Brodmann. Journal für Psychologie und Neurologie 1918;24:1-10 